\title{
15 YEARS OF AQUA MODIS ON-ORBIT OPERATION, CALIBRATION, AND PERFORMANCE
}

\author{
X. Xiong ${ }^{1}$, A. Angal ${ }^{2}$, A. Wu ${ }^{2}$, Z. Wang ${ }^{2}$, W. Barnes ${ }^{3}$, and V. Salomonson ${ }^{4}$ \\ ${ }^{1}$ Sciences and Exploration Directorate, NASA/GSFC, Greenbelt, MD 20771, USA \\ ${ }^{2}$ Science Systems and Applications Inc., 10210 Greenbelt Road, Lanham, MD 20706, USA \\ ${ }^{3}$ University of Maryland, Baltimore County, 1000 Hilltop Circle, Baltimore, MD 21250, USA \\ ${ }^{4}$ University of Utah, Salt Lake City, UT 84112, USA
}

\begin{abstract}
Since launch on May 04, 2002, Aqua MODIS has successfully operated for 15 years and continuously produced from its observations many data products in support of a broad range of scientific research activities and applications. Its overall mission success has relied heavily on the dedicated efforts to operate and calibrate the instrument and to track and correct on-orbit changes in sensor responses. This paper provides an overview of Aqua MODIS instrument operation and calibration activities, algorithm improvements, and look-up-table (LUT) updates. Results derived from various on-orbit calibration targets are presented to demonstrate sensor long-term performance. Also discussed in this paper are challenging issues identified and future efforts to maintain instrument calibration and data quality.
\end{abstract}

Index Terms - Aqua, MODIS, sensor, calibration

\section{INTRODUCTION}

The MODIS is one of the key instruments developed for the NASA's EOS missions, currently operated on the Terra and Aqua spacecraft. Since launch in May 2002, the Aqua MODIS has successfully operated for 15 years and produced many high quality long-term data records, enabling support for a broad range of research activities and applications by the science and user community [1-3]. MODIS observations are made using 36 spectral bands with wavelengths from 0.41 to $14.5 \mu \mathrm{m}$. Bands 1-19, and 26 are the reflective solar bands (RSB), covering wavelengths from 0.41 to 2.3 $\mu \mathrm{m}$, and the remaining bands $20-25$ and 27-36, from 3.7 to $14.5 \mu \mathrm{m}$, are referred to as the thermal emissive bands (TEB). MODIS instrument was equipped with a complete set of on-board calibrators (OBC), which include a solar diffuser (SD), a solar diffuser stability monitor (SDSM), a v-groove blackbody (BB), a spectroradiometric calibration assembly (SRCA), and a space view (SV) port, capable of performing its onorbit radiometric, spatial, and spectral calibration and characterization [4]. In addition, regularly scheduled lunar observations and data selected from well characterized ground targets are used to support RSB calibration, especially for the sensor's response versus scan-angle (RVS) [5-7]. This paper provides an overview of Aqua MODIS mission-long operation and calibration activities, its level 1B (L1B) algorithm enhancements, and LUT updates. It illustrates OBC on-orbit performance and changes in sensor responses. Remaining calibration challenges and strategies for future improvements are also discussed.

\section{SENSOR OPERATION AND CALIBRATION}

The MODIS Characterization Support Team (MCST) at NASA/GSFC is responsible for MODIS instrument operations and calibration, monitoring its on-orbit performance and tracking changes in sensor responses. It is also responsible for L1B algorithm enhancements and LUT updates in order to maintain MODIS data quality. For Aqua MODIS, the same operational configuration has been used through its entire mission. As an effort to characterize instrument performance and to assure MODIS data quality, various calibration activities have been performed on a regular basis, 
including SD and SDSM calibration events, BB warmup and cool-down (WUCD), SRCA operation in three different modes, electronic calibrations (ECAL), and lunar observations. Summarized in Table 1 are different calibration activities performed for the Aqua MODIS.

Table 1 Aqua MODIS On-orbit Calibration Activities (as of May 1, 2017)

\begin{tabular}{|c|c|}
\hline SD/SDSM & 595 \\
\hline BB WUCD & 64 \\
\hline SRCA (Radiometric) & 178 \\
\hline SRCA (Spatial) & 69 \\
\hline SRCA (Spectral) & 45 \\
\hline ECAL & 74 \\
\hline Lunar Observations & 147 \\
\hline
\end{tabular}

The SD/SDSM and lunar observations are primarily used for RSB calibration and the BB for TEB. Depending on specific applications, some calibration activities have been performed more frequently than others. As mission continues, some of the calibration frequencies have been gradually reduced in response to degradation experienced by the instrument and considerations to further extend its lifetime. Data collected from various calibration activities are analyzed by MCST to assess instrument performance, to determine changes in sensor responses, and, most importantly, to derive and update calibration LUTs.

\section{PERFORMANCE}

From launch to present, Aqua MODIS has continued to operate normally in a stable environment. This is illustrated in Figure 1 by the instrument temperature mission-long trending. Since launch, on-orbit changes have been less than $2.5 \mathrm{~K}$ with most of the changes occurring during the first few years. The temperatures of the VIS and NIR focal plane assembly (FPA) have experienced similar changes. At mission beginning, the cold (SMIR and LWIR) FPA temperatures were effectively controlled at $83 \mathrm{~K}$ (see Figure 2). Starting from approximately 2008, the temperatures have gradually seen small variations with the maximum peak in 2013 (up to $0.5 \mathrm{~K}$ on average). This increase is largely due to loss of its radiative cooler margin. Initially, constant calibration coefficients (via LUTs) were used for several LWIR spectral bands during BB
WUCD when the BB temperature is above their saturation limits. To minimize the impact due to focal plane temperature variations on calibration coefficients during BB WUCD, the TEB calibration algorithm was modified in Collection 6 by applying temperature dependent coefficients. For Aqua MODIS, the BB temperatures are nominally controlled at $285 \mathrm{~K}$. As shown in Figure 3, its performance has been extremely stable. The long-term drift and seasonal variations are well within $10 \mathrm{mK}$.

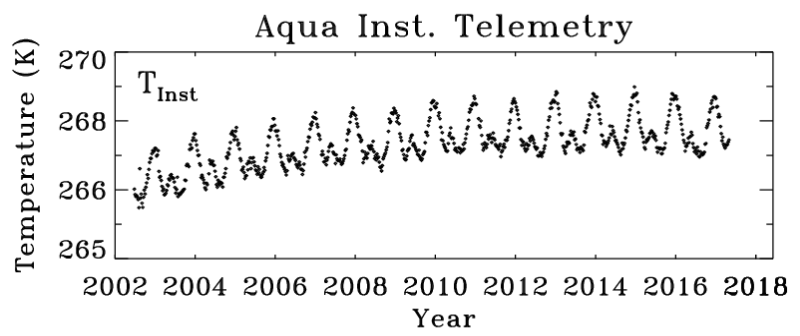

Figure 1 Aqua MODIS instrument temperatrures

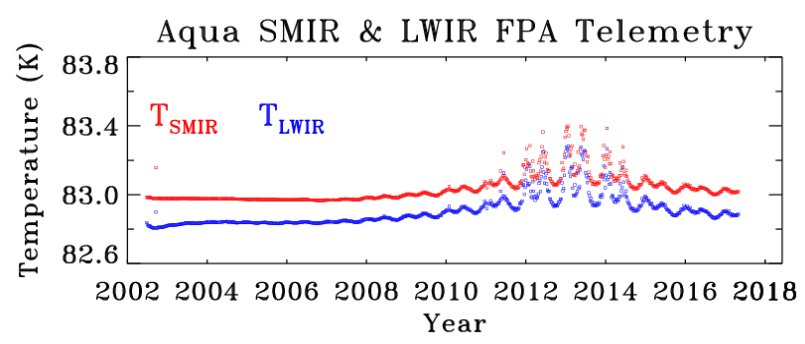

Figure 2 Aqua MODIS cold FPA tempertures

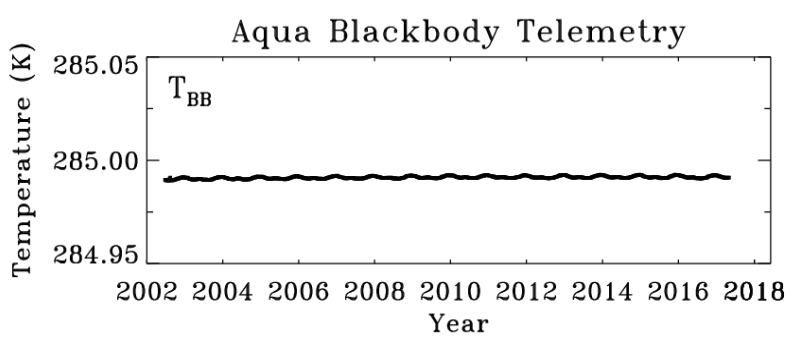

Figure 3 Aqua on-board BB temperatures

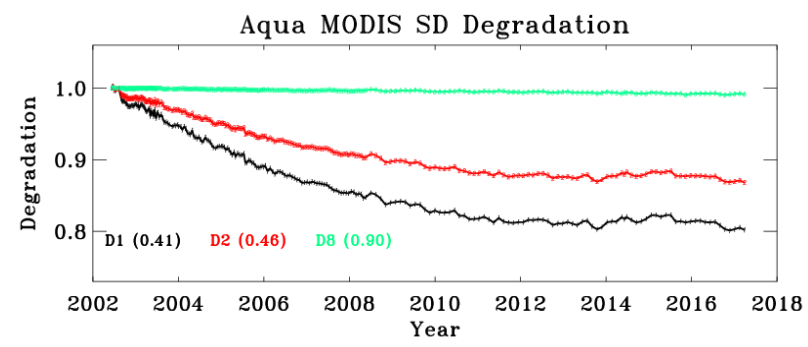

Figure 4 Aqua MODIS solar diffuser (SD) on-orbit degradation at selected wavelengths $(0.41,0.46$, and 0.90 $\mu \mathrm{m})$. 
The SD on-orbit degradation is regularly tracked by the SDSM that has 9 individual detectors (D1-D9), covering wavelengths from $0.4-0.94 \mu \mathrm{m}$. The longterm SD degradation derived from SDSM is presented in Figure 4, showing larger changes at shorter wavelengths. The similar wavelength dependent degradation has also been observed in Terra MODIS and S-NPP VIIRS [8].

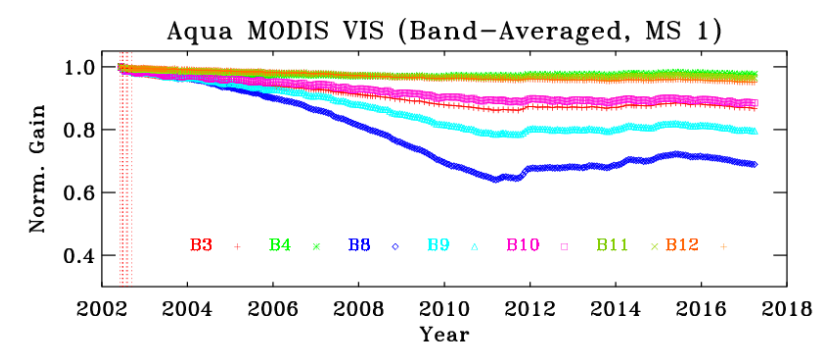

Figure 5 Normalized gains for Aqua MODIS VIS bands

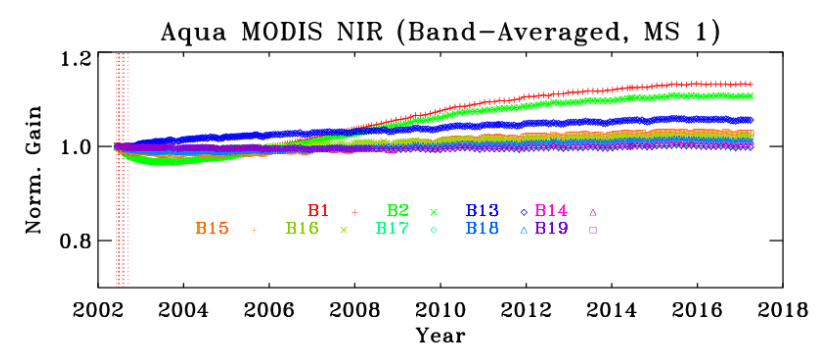

Figure 6 Normalized gains for Aqua MODIS NIR bands

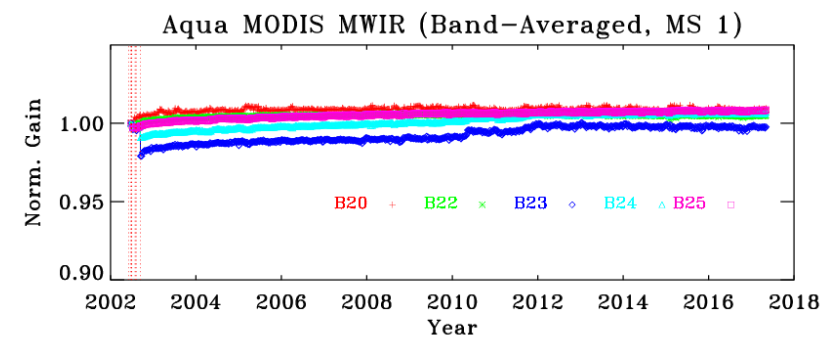

Figure 7 Normalized gains for Aqua MODIS MWIR bands

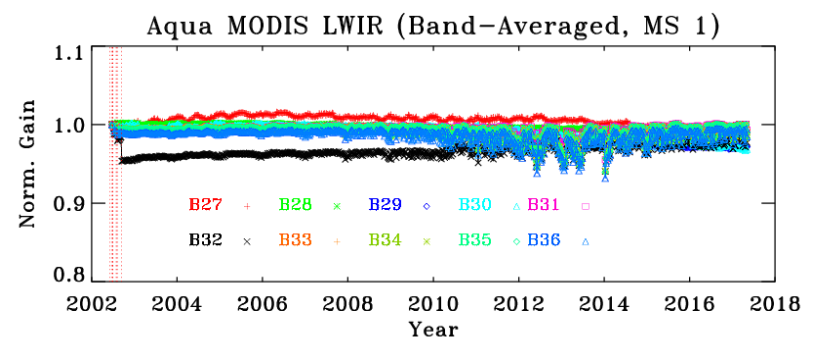

Figure 8 Normalized gains for Aqua MODIS LWIR bands

Illustrated in Figures 5 and 6 are on-orbit changes in Aqua MODIS responses (gains) for the VIS and NIR spectral bands (band averaged, mirror side 1). Clearly, several short wavelength VIS bands have experienced large gain changes. Results in Figures 5 and 6 are derived from SD observations. Similar trends have also been observed based on lunar calibrations. The SD and lunar observations are made at different scan angles. Their responses and that from selected EV targets at various scan angles have been used to characterize sensor's RVS. Compared to VIS and NIR spectral bands, the changes in SWIR band responses have been much smaller (less than $2 \%$ ).

In general, the changes for MWIR and LWIR spectral band responses are very small. Their mission-long trends are presented in Figures 7 and 8. Noticeable variations in LWIR spectral band responses, starting from around 2008, are clearly correlated to the cold FPA temperature variations. It should be pointed out that the TEB calibration is performed on a scan-byscan basis, except for a few bands during part of the BB WUCD, the calibration impact due to FPA temperature variations are negligible [9].

Overall instrument spatial and spectral performance, tracked using routine SRCA measurements, has been very stable through its entire mission. An issue identified pre-launch for Aqua MODIS was coregistration offsets between warm and cold FPA. As a result, all bands on the warm FPA failed to meet the band-to-band registration $(\mathrm{BBR})$ requirements $( \pm 0.1$ $\mathrm{km}$ ) with any of the bands on the cold FPA, and vice versa. These offsets remained on-orbit. For most VIS and NIR bands, the changes in their center wavelengths and bandwidths are small, less than 0.5 and $1.0 \mathrm{~nm}$, respectively. Due to its lamp degradation, the SRCA operation configuration, procedure, and frequency have been modified for a few times over the years. At launch, 15 detectors (13 in band 6) were identified as inoperable and 3 detectors were noisy. Since then, there have been no new inoperable detectors. The number of noisy detectors has increased to 10 with no change in the last two years. All new noisy detectors are in the TEB.

\section{CHALLENGES AND IMPROVEMENTS}

Launched in May 2002, Aqua MODIS has operated significantly beyond its design lifetime of 6 years. Though instrument operation conditions have been very stable over time, large scan mirror degradation 
experienced on-orbit has led to changes in sensor's RVS and potentially its polarization sensitivity. This was the case in Terra MODIS. At the beginning of C6, only a few ocean color bands in Aqua MODIS need to apply earth view data to derive their RVS. Recently, bands 1-4 have also used the EV response trending to improve their RVS characterization and calibration updates [10]. If necessary, same strategy and approach will be extended to other spectral bands.

Large SD degradation (approximately 20\% at 0.41 $\mu \mathrm{m})$ in its reflectance property, though constantly monitored and corrected, could potentially impact the calibration quality at short wavelengths. This needs to be continuously monitored and dealt with accordingly. Since SDSM only covers wavelengths up to $0.94 \mu \mathrm{m}$, any potential SD degradation at longer wavelengths in the SWIR region must be carefully examined and corrected if necessary.

As illustrated in Figure 2, the cold FPA temperature variations have gradually decreased in the last few year, as a result of slow recovery of sensor radiative cooler performance. For future improvements, MCST has started a comprehensive assessment of the crosstalk impact on Aqua MODIS TEB bands and/or detectors [11]. This is similar to the work performed for Terra MODIS LWIR PV bands [12]. In addition to crosstalk coefficients, the correction algorithms will be developed and applied if necessary in the L1B production. Various lessons learned since launch, including those from Terra MODIS on-orbit operation and calibration, will be considered for future improvements of Aqua MODIS on-orbit performance.

\section{ACKNOWLEDGEMENTS}

Authors would like to acknowledge contributions and technical support provided by other MCST members.

\section{REFERENCES}

[1] Salomonson, V., W. Barnes, X. Xiong, S. Kempler, and E. Masuoka, "An Overview of the Earth Observing System MODIS Instrument and Associated Data Systems Performance," IEEE International Geoscience and Remote Sensing Symposium (IGARSS), pp. 970-972, 2002
[2] Xiong, X., B. Guenther, A. Angal, W. Barnes, V. Salomonson, J. Sun, and B. Wenny, "MODIS radiometric calibration program, methods and results," IEEE

International Geoscience and Remote Sensing Symposium (IGARSS), pp. 6924-6927, 2012

[3] Xiong, X., M. King, V. Salomonson, W. Barnes, B. Wenny, A. Angal, A. Wu, S. Madhavan, D. Link, "Moderate Resolution Imaging Spectroradiometer on Terra and Aqua Missions," Optical Payloads for Space Missions (ed S.-E. Qian), John Wiley \& Sons, Ltd, Chichester, UK. doi: 10.1002/9781118945179, 2015

[4] Xiong, X., K. Chiang, J. Esposito, B. Guenther and W.L. Barnes, "MODIS On-orbit Calibration and Characterization," Metrologia 40, 89-92, 2003

[5] Sun, J., X. Xiong, W. Barnes, and B. Guenther, "MODIS Reflective Solar Bands On-orbit Lunar Calibration," IEEE TGRS, Vol. 45, No. 7, 2383-2393, 2007

[6] Sun, J., X. Xiong, A. Angal, H. Chen, A. Wu, and X. Geng, "Time-Dependent Response Versus Scan Angle for MODIS Reflective Solar Bands", IEEE TGRS, vol. 52, issue 6, pp. 3159-3174, June 2014

[7] Chen, H., X. Xiong, A. Angal, X. Geng, and A. Wu, "Alternative method of on-orbit response-versus-scan-angle characterization for MODIS reflective solar bands", Journal of Applied Remote Sensing, vol. 10(2), 024004, 2016

[8] Xiong, X., J. Butler, K. Chiang, B. Efremova, J. Fulbright, N. Lei, J. McIntire, H. Oudrari, Z. Wang, and A. $\mathrm{Wu}$, "Assessment of S-NPP VIIRS On-Orbit Radiometric Calibration and Performance," Remote Sens. 2016, 8(2), 84; doi: $10.3390 / \mathrm{rs} 8020084$

[9] Xiong, X., A. Wu, B. N. Wenny, S. Madhavan, Z. Wang, Y. Li, N. Chen, W. Barnes, and V. Salomonson, "Terra and Aqua MODIS Thermal Emissive Bands On-Orbit Calibration and Performance," IEEE TGRS, vol. 53, Issue 10, pp. 5709 - 5721, 2015

[10] Angal, A., X. Xiong, A. Wu, H. Chen, X. Geng, D. Link, Y. Li, A. Wald, and J.. Brinkmann, "On-orbit Performance and Calibration Improvements for the Reflective Solar Bands of Terra and Aqua MODIS", SPIE Proceedings, Vol. 9881, 2016

[11] Keller, G., Z. Wang, A. Wu, and X. Xiong, "Aqua MODIS band 24 crosstalk striping," IEEE GRSL, vol. 14, no. 4, pp. 475-479, 2017

[12] Wilson T., A. Wu, X. Geng, Z. Wang, X. Xiong, "Analysis of the electronic crosstalk effect in Terra MODIS long-wave infrared photovoltaic bands using lunar images," SPIE Proceedings, Vol 10004, 2016 